\title{
Design of Self-Reconfigurable Multiarm Robot Mechanism Based on Deployable Kinematic Chains
}

\author{
Fu-Qun Zhao', Sheng Guo ${ }^{*}$, Hai-Jun Su², Hai-Bo Qu ${ }^{1}$ and Ya-Qiong Chen ${ }^{1}$
}

\begin{abstract}
As the structures of multiarm robots are serially arranged, the packaging and transportation of these robots are often inconvenient. The ability of these robots to operate objects must also be improved. Addressing this issue, this paper presents a type of multiarm robot that can be adequately folded into a designed area. The robot can achieve different operation modes by combining different arms and objects. First, deployable kinematic chains (DKCs) are designed, which can be folded into a designated area and be used as an arm structure in the multiarm robot mechanism. The strategy of a platform for storing DKCs is proposed. Based on the restrictions in the storage area and the characteristics of parallel mechanisms, a class of DKCs, called base assembly library, is obtained. Subsequently, an assembly method for the synthesis of the multiarm robot mechanism is proposed, which can be formed by the connection of a multiarm robot mechanism with an operation object based on a parallel mechanism structure. The formed parallel mechanism can achieve a reconfigurable characteristic when different DKCs connect to the operation object. Using this method, two types of multiarm robot mechanisms with four DKCs that can switch operation modes to perform different tasks through autonomous combination and release operation is proposed. The obtained mechanisms have observable advantages when compared with the traditional mechanisms, including optimizing the occupied volume during transportation and using parallel mechanism theory to analyze the switching of operation modes.
\end{abstract}

Keywords: Design, Multiarm robot, Deployable kinematic chain, Parallel mechanism, Motion set

\section{Introduction}

The multiarm robot mechanism is a type of mechanism that is composed of several kinematic chains in a serial connection. The typical multiarm robot mechanisms are structurally similar and include a multiarm cooperative operation mechanism, space manipulator, certain multifingered hand mechanisms, and a walking robot [1-7]. The finger or leg of the multifingered or walking robot mechanism can be treated as the arm of the multiarm robot mechanism. As these types of mechanisms can be utilized in several applications such as fire rescue, satellite coupling, and motion object capture, the robot

\footnotetext{
${ }^{*}$ Correspondence: shguo@bjtu.edu.cn

${ }^{1}$ School of Mechanical, Electronic and Control Engineering, Beijing

Jiaotong University, Beijing 100044, China

Full list of author information is available at the end of the article
}

mechanism has attracted considerable attention. In recent years, certain scholars have attempted to improve the performance of the multiarm robot mechanism by optimizing the design mechanism structure and have proposed a novel conceptual design theory with significant results $[8,9]$.

However, the traditional multiarm robot mechanism often suffers from certain key problems. Generally, the arms of these robots have links that are connected in series. Because the mechanism requires a higher degree of freedom (DOF), it usually requires multiple actuation joints to connect each link in series. Consequently, the body of the mechanism has too many links and relatively large size. The large size of the body causes inconvenience during the transportation of these types of robots. Therefore, it is necessary to design a stacking method for the mechanism so that it can be reasonably contained in a 
carrier when it is transported or when it is not functioning and can be easily expanded when it must be operated. Based on the structural determination of the mechanism, the achievable operations should be diversified as much as possible to improve work efficiency. For example, when the mechanism is applied for the exploration of the moon and in deep space, it is often necessary for the mechanism to clean, pick up, transport, and load the target after close observation and analysis. Sometimes, observation or detection of the target for grinding, drilling, and other operations is also required. As the mechanism can only perform deterministic tasks after finalizing the structural design, to achieve diversified operations, it is necessary to use the multiarm concept to obtain a reasonable switch to achieve a change in the working mode.

When the multiarm robot mechanism connects to the operation object, the formed structure can be treated as a parallel structure; several scholars use the design concept of the parallel mechanism to optimize the operation configuration of such a mechanism. The purpose is to change a single-arm operation into a multiarm operation and allocate the required number of motors to avoid creating a significantly long single arm. In addition, this method considers the operation object as the moving platform or output of the formed virtual parallel mechanism. Consequently, such a mechanism can perform deterministic operation tasks. Based on the concept of a parallel mechanism, Özgür et al. $[10,11]$ designed a dexterous hand and obtained a new type of grasping mechanism. Borràs et al. $[12,13]$ also designed a dexterous hand by using the parallel mechanism concept and analyzed the dexterous hand through the force-closure method. The kinematics problem of the dexterous hand is solved by analyzing a Jacobian matrix of the formed parallel mechanism. Ding et al. [14] and Zhai et al. [15] designed an integrated arm and leg mechanism that can traverse complex terrains and has redundant degrees of freedom. Corves et al. [16] designed a robot system with reconfigurable properties by using the design idea of a parallel mechanism, which can achieve repetitive grabbing of objects and the reconstruction of dynamic characteristics. Nefz et al. [17] proposed a multifinger mechanism for gripping. By using the method where the fingers and objects can form a parallel mechanism, the appropriate manipulator is obtained. The corresponding performance was investigated. To improve the performance of the walking robot, Ding et al. [14] proposed a wheel-legged hexapod robot to manipulate objects with legs. This robot mimics the control and movement of insects pertaining to their legs. Koyachi et al. [18] presented a concept of "limb mechanism," which consists of links and joints that interface the body of the robot with the ground. Inoue et al. [19] presented an approach to improve the maneuverability of the arm by adjusting the motion of the legs. To improve the operation ability of multiarm robots, several scholars have conducted research on capture characteristics [20, 21], reconfigurable manipulation [22], modeling [7], and performance evaluation [23].

As mentioned above, another key problem is in designing a stacking method through which the mechanism can be satisfactorily contained in a carrier when it is transported or has stopped working, and it can be easily expanded during the work. Inspired by the characteristics of the traditional deployable mechanism, the multiarm robot mechanism can be designed as a mechanism with a deployable arm structure. The characteristics of the traditional deployable mechanism include small volume for easy storage, ease in transportation, and certain other performance characteristics. The mechanism can fold into designated areas during transportation and unfold when it is required for work. The design procedure of the traditional deployable mechanism is to first design individual deployable units [24-26], and then combine these units to form a deployable mechanism. To create a traditional multiarm robot mechanism with deployable performance, we can reference the design method of the traditional deployable mechanism. On the one hand, the arms can be designed as deployable units, which can be folded into a designated area. On the other hand, the multiarm robot mechanism and operation object or external environment can constitute a parallel mechanism structure. Therefore, the combination principle of deployable units can be achieved by the parallel mechanism synthesis method. The obtained novel multiarm robot mechanisms possess advantages such as ease in transportation and easy configurability of operations when compared to traditional multiarm robot mechanisms.

Hence, this research studied the design of deployable kinematic chains (DKCs), which can be folded into a designated area and be used for the design of the robot. This method is aimed at introducing deployable characteristics and is a design method that incorporates parallel mechanism into the traditional multiarm robot mechanism so that the arms of the robot can be easily deployed and demonstrate improved storage performance. At the same time, the structural synthesis for operational task topology can be performed through the concept that the multiarm robot mechanism and the operating object can form a structure with a parallel mechanism. This part is presented in Section 2. The strategy of a platform for storing DKCs is proposed. In Section 3, a class of DKCs called base assembly library (BAL) is discussed and an assembly method for the design of the multiarm robot is presented. Using this method, two types of robot mechanisms with four DKCs that can switch operating modes 
to complete different tasks through autonomous combination and release with operation are presented in Section 4. The advantages of the proposed mechanisms when compared to the traditional mechanisms are demonstrated. Finally, the conclusion is drawn in Section 5.

\section{Conceptual Design of Multiarm Robot}

\subsection{Description of Multiarm Robot Working Mode and Task} Topology

In this paper, two types of multiarm robot mechanisms are proposed, which can be applied to perform different operations. The first type of mechanism is the TypeI mechanism. Its topological logic diagram and working mode are shown in Figure 1. Figure 1a shows that each DKC is folded into its designated area in the fixed platform when it is nonworking. If the mechanism is required to operate an object, some of the DKCs will unfold and connect to the object as shown in Figure 1b. It is easy to observe that a parallel mechanism structure will be formed when two or more DKCs connect to the object. Those DKCs are limbs of the virtual parallel mechanism, and the object is a moving platform in this parallel mechanism. Therefore, different operation modes can be achieved when different DKCs connect to the object. In this case, the formed parallel mechanism can also be treated as a reconfiguration. Here, we define the chain that connects to the object as an active DKC and the nonworking chain as a resting DKC.

In the folded-deployed process, every joint of the chain is an actuated joint. When DKCs connect to the object and perform operations on the object, some of the joints will turn into passive joints based on the parallel mechanism theory.

The second type of mechanism is the Type-II mechanism, as shown in Figure 2. This mechanism has a twolevel platform, which includes the upper and lower platforms for storing DKCs. Both the upper and lower platforms can connect to the object to construct a parallel mechanism through DKCs. When the multiarm robot mechanism is used as a spatial manipulator to perform operational tasks, the two platforms are fixed to a fixed base. The task topological logic diagram of the Type-II mechanism is shown in Figure $2 \mathrm{~b} . \mathrm{PM}_{1}$ is composed of object- 1 and the DKCs of the upper platform, and $\mathrm{PM}_{2}$ is composed of object-2 and DKCs of the lower platform. Each parallel mechanism structure can produce

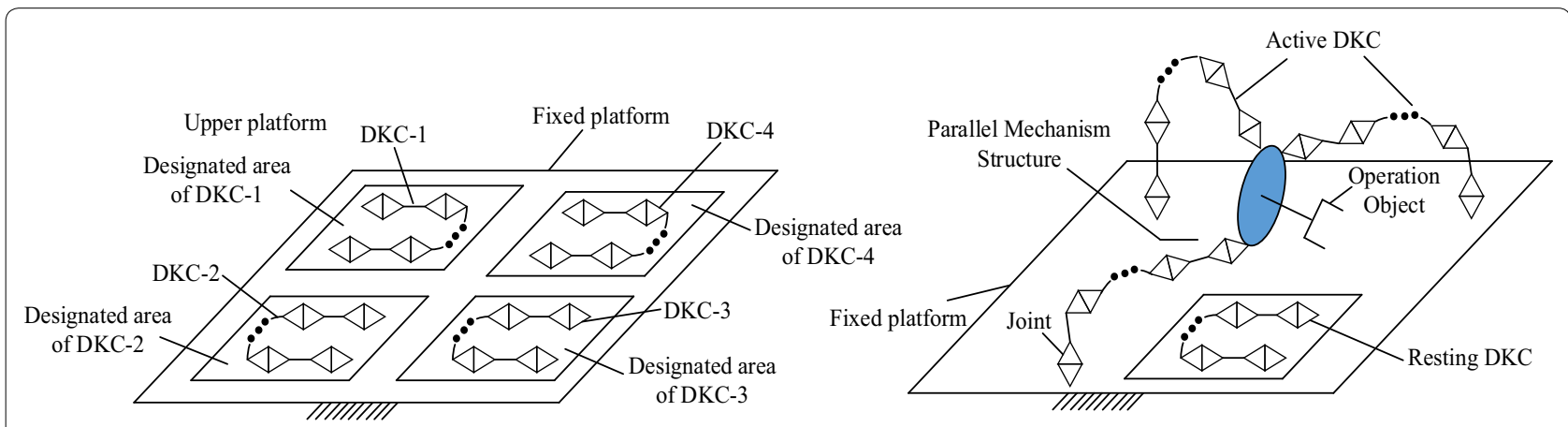

a Each DKC is folded into a designated area

b Topological logic diagram in working state

Figure 1 Topological logic diagram and working mode of the Type-I mechanism

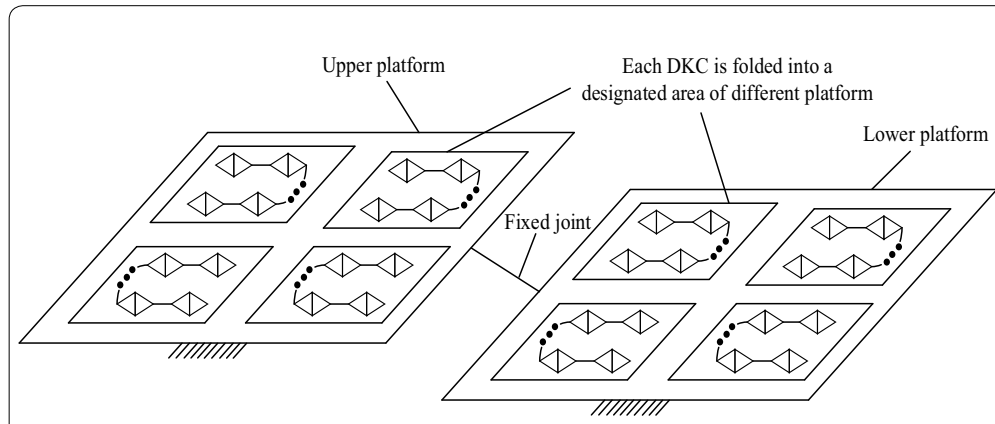

a Each DKC is folded into a designated area in different platforms

Figure 2 Task topological logic diagram of the Type-II mechanism

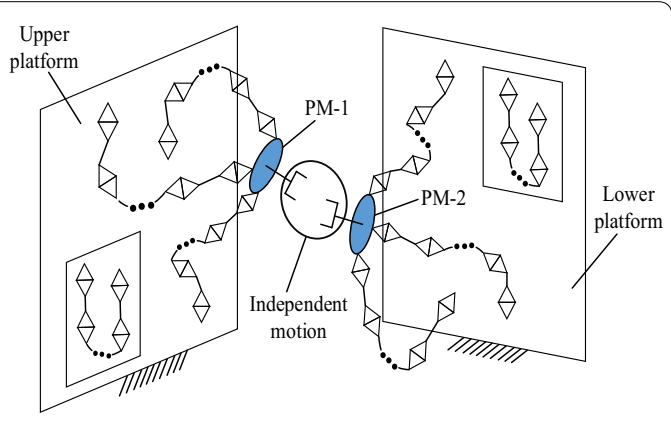

b Topological logic diagram in working state 
independent motion. For this type of mechanism, two parallel mechanisms can achieve concurrent operation to achieve superposition and complementarity of the kinematic performance.

If the operation object is the ground, the Type-I mechanism can be used as a walking robot mechanism. When it works, each chain can expand and form a parallel mechanism with the ground. The fixed platform will become the end effector of the virtual parallel mechanism, while the ground will become the fixed platform of this virtual parallel mechanism. The mechanism can achieve the task requirements of walking and carrying a load. In the TypeII mechanism, the upper and lower platforms can also construct a configuration in the form of "serial-parallel" structure through the DKCs that expand based on the external environment and operating objects. When the DKCs of the lower platform connect to the ground, the formed parallel mechanism can adjust the posture and other work tasks of the upper platform.

It should be noted that there are various types of contacts between the end of the walking robot or multifinger hand mechanism and the operation object; therefore, the relative DOFs between the end of these mechanisms and the operation object can range from 0 to 6 . To clearly illustrate the application of the parallel mechanism design idea into the multiarm robot mechanism design, this paper primarily considers the mechanism that can be applied to the spatial manipulator. Both the joint of the chain and the contact between the chains and the object indicate one-DOF motions. Nevertheless, the contact type considered in this paper can also be used as a special form of the multiple contact forms of terminal contact in a walking robot mechanism.

\subsection{Configurable Operation Degree of Multiarm Robot}

After proposing the concept of the multiarmed robot mechanism, it is necessary to determine the number of DKCs configured by the mechanism and the DKC switching strategy to perform different operation modes. The number of DKCs to be configured in each platform should be greater than two and less than six for the following reasons:

1. To form a closed-loop parallel mechanism structure between the DKCs and an object, the number of DKCs should not be less than two.

2. As the maximum DOF of an object in space is six, the virtual parallel mechanism formed by the multiple arms and object should have a maximum of six DKCs for nonredundancy, that is, the maximum number of DKCs configured in the platform is six.

As mentioned in Section 2.1, the DKC that connects to the object is an active DKC, denoted by A. The DKC that is nonworking is a resting DKC, denoted by $\mathrm{R}$, as listed in Table 1. $\mathrm{L}$ indicates the number of DKCs that connect to the object. The multiarm robot mechanism can achieve different operating modes on an object by connecting different DKCs to the object. Because the structures of the DKCs are different, the topological forms of parallel mechanisms formed by the different DKCs and object are different, which results in different operations on the object. After determining the number of DKCs in the platform, the total number of operation modes that the DKCs can implement on an object is denoted by a configurable operation degree. Then, the relation between the DOF of the entire mechanism, configurable operation degree, and number of DKCs can be obtained as listed in Table 1.

\subsection{Storage Area for Deployable Kinematic Chain (DKC) of Multiarm Robot}

Because the shape or length of DKC is often restricted by the dimension of the storage area, the area for DKC storage should be determined first before designing the DKC configuration. Further, as the number of DKCs increases, the storage area of each DKC should be smaller.

Figure 3 shows the storage area diagrams of the most common six-arm and four-arm mechanisms. In a symmetrical arrangement of chains, six chains divide $360^{\circ}$ into six sections. Similarly, four chains divide $360^{\circ}$ into four sections. Assuming that the base link is the first link of each DKC and is connected to the folding platform, the minimum space occupied by each DKC after

Table 1 Degree of freedom, kinematic chain number, and the degree of configurable operation $\left(\lambda_{m}\right)$

\begin{tabular}{|c|c|c|c|c|c|c|c|}
\hline \multirow[t]{2}{*}{ L } & \multicolumn{7}{|l|}{$\mathbf{R}$} \\
\hline & 0 & 1 & 2 & 3 & 4 & 5 & 6 \\
\hline 2 & $\mathrm{DOF} \leq 2 ; \lambda_{\mathrm{m}}=1$ & - & - & - & - & - & - \\
\hline 3 & $\mathrm{DOF} \leq 3 ; \lambda_{\mathrm{m}}=1$ & $\mathrm{DOF} \leq 2 ; \lambda_{\mathrm{m}}=3$ & - & - & - & - & - \\
\hline 4 & $\mathrm{DOF} \leq 4 ; \lambda_{\mathrm{m}}=1$ & $\mathrm{DOF} \leq 3 ; \lambda_{\mathrm{m}}=4$ & $\mathrm{DOF} \leq 2 ; \lambda_{\mathrm{m}}=6$ & - & - & - & - \\
\hline 5 & $\mathrm{DOF} \leq 5 ; \lambda_{\mathrm{m}}=1$ & $\mathrm{DOF} \leq 4 ; \lambda_{\mathrm{m}}=5$ & $\mathrm{DOF} \leq 3 ; \lambda_{\mathrm{m}}=10$ & DOF $\leq 2 ; \lambda_{m}=10$ & - & - & - \\
\hline 6 & $\mathrm{DOF} \leq 6 ; \lambda_{\mathrm{m}}=1$ & DOF $\leq 5 ; \lambda_{m}=6$ & DOF $\leq 4 ; \lambda_{m}=15$ & DOF $\leq 2 ; \lambda_{m}=20$ & DOF $\leq 2 ; \lambda_{m}=15$ & - & - \\
\hline
\end{tabular}




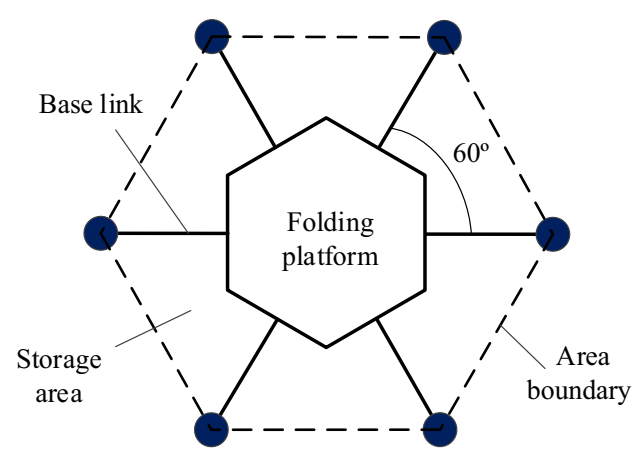

a Storage area of six-arm mechanism

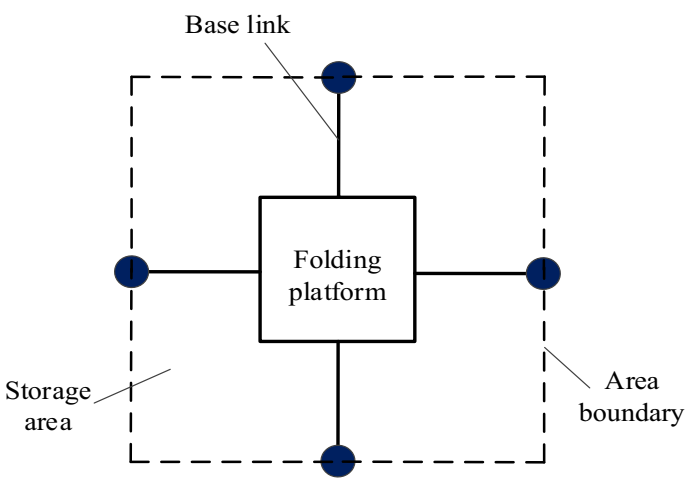

b Storage area of four-arm mechanism

Figure 3 Storage area of the mechanisms with six and four arms

folding the design is never less than that occupied by the base link. Therefore, if the multiarm robot mechanism is designed to occupy the minimum area in the folding state, the minimum occupied space of the mechanism in the folding state is composed of the folding platform and each base link. Thus, each base link forms both the storage area and area boundary as shown in Figure 3.

To achieve the spatial operation of DKCs on an object, each DKC has more than three links. Frequently, the shape of the link is a straight line. At the same time, the storage area of the four-arm mechanism is decomposed into four rectangular or square areas, which can better store each DKC and improve the utilization efficiency. Because the mechanism usually does not require six DOFs when performing a single task, the four-arm robot mechanism is sufficient to integrate multiple tasks by using different DKCs to connect to the object.

Therefore, we designed the configuration of the DKC based on the region and dimension constraints of the rectangle and square in the four-arm robot mechanism. The designed DKC configuration can fold into the rectangular storage area in the nonworking state and is in the same plane as the folding platform. By connecting different DKCs to an object, the virtual parallel mechanism can be reconfigured, which can change the operation mode of the multiarm robot mechanism.

According to Table 1, when four DKCs are configured in the folding platform, the number of active and resting chain configurations are: (A:4, R:0), (A:3, R:1), and $(A: 2, R: 2)$. Let us consider the case of (A:3, R:1) as an example. The object can connect with three limbs to form a parallel structure as shown in Figure 4a. In Table 1 , when $A=3$ and $R=1$, four combinations will appear as shown in Figure 4. In the nonredundant parallel mechanism, the three limbs can give the object three DOFs. Here, we define these three DOFs as the initial DOFs. As different limbs have different constraints on the object, the $\mathrm{PM}_{1}$ structure formed by the object and limbs-1,2,3 changes into the $\mathrm{PM}_{2}$ structure formed by the object and limbs-1, 2, 4, which can modify at least one DOF. Similarly, the $\mathrm{PM}_{3}$ and $\mathrm{PM}_{4}$ structures can also modify one DOF from the previous configuration. Therefore, the four-arm robot mechanism can have the operation of all six DOFs on the object by using three different limbs.

Figure 5a shows the boundary of each storage area and the space occupied by the mechanism when it is folded. Because the DKC configuration is composed of a base link in series with several links, the storage area can be divided into two subareas. One is the storage area of the base link. The other is the additional area of the rest of the DKC. In the four-arm robot mechanism, the lengths of the four base links determine the boundary of the occupied space. As shown in Figure 5, base link- 1 and base link- 3 determine the length of occupied space along the $x$-axis. Similarly, base link-2 and base link-4 determine the length of occupied space along the $y$-axis. In addition, each base link determines the length in one direction of its adjacent region. Therefore, the storage area (region within the dotted lines) for each DKC is formed as shown in Figure $5 \mathrm{a}$. Figure $5 \mathrm{~b}$ is a case of the four-arm robot mechanism that shows the folding configuration and the corresponding definition.

The advantages of this arrangement are as follows. First, the rectangular region can be used to store the DKC to the maximum extent. Besides, when the mechanism acts as a spatial manipulator or multifingered hand, it can switch the operation mode using different DKCs to connect to the operation object. In the next section, we discuss how to design the DKC 


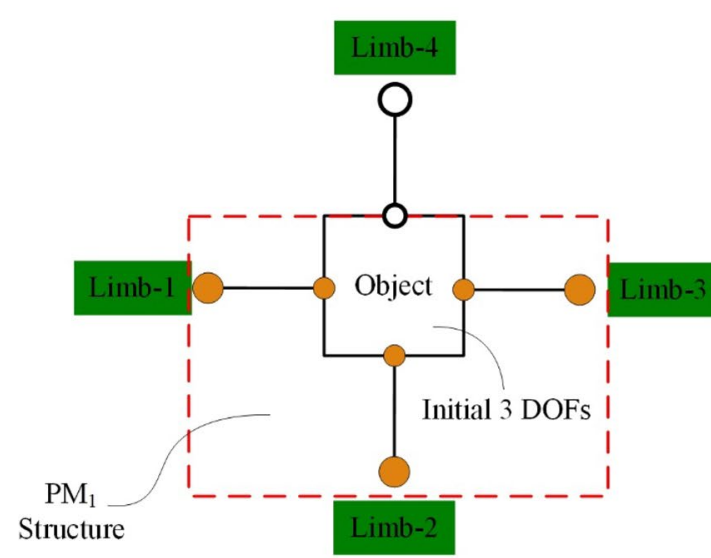

a Object connects to limbs- $1,2,3$

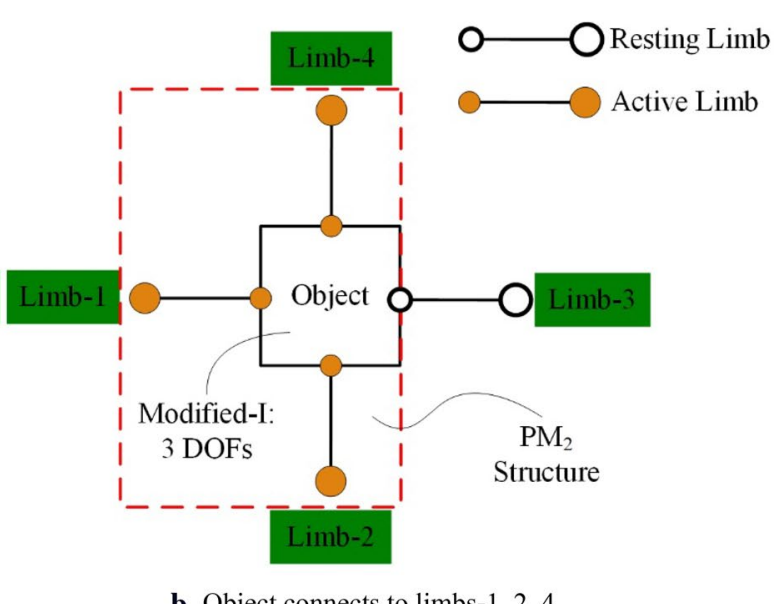

b Object connects to limbs-1, 2, 4

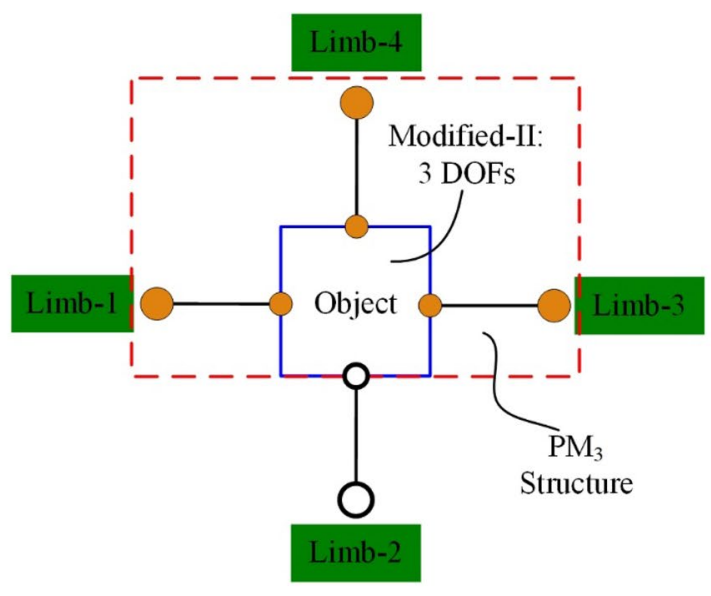

c Object connects to limbs-1, 3, 4

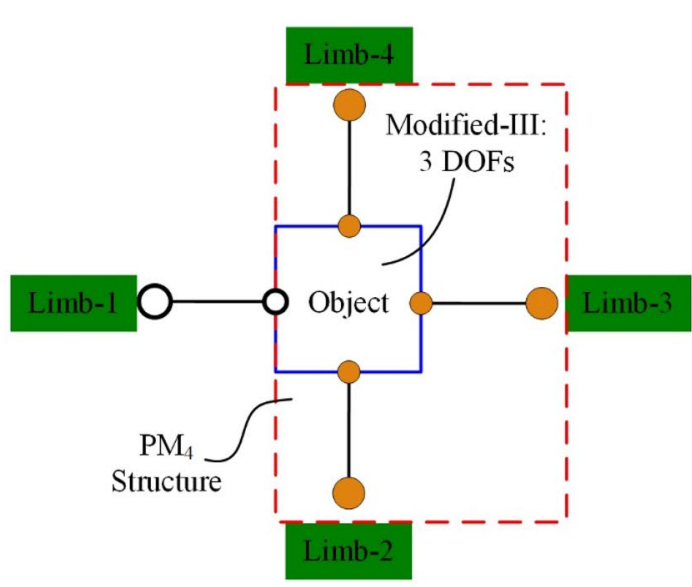

d Object connects to limbs-2, 3, 4

Figure 4 Switch strategy of limbs in the four-arm mechanism

configuration for the four-arm robot mechanism under the restriction of a rectangular region.

\section{Configuration Design of DKC}

\subsection{Determination of Joint Number}

Frequently, if the operation of $F$ DOFs is performed on the operation object, the link degree of each chain must be at least equal to $F$. In the nonredundant case, the link degree $C_{i}$ should be no more than six; then:

$$
6 \geq C_{i} \geq F,
$$

where the link degree $C_{i}$ is equal to the sum of DOFs of the joints in each chain.

The joint number of the kinematic chain is denoted by $m_{i}$. Hence, we obtain:

$$
6 \geq m_{i} \geq F .
$$

According to Table 1, the number of DOFs of the mechanism is limited, that is:

$$
6 \geq F \geq 2 .
$$

Assuming the DOFs of all joints are equal to $1, f_{i}=1$. Then, from Eq. (2), it can be determined that the number of joints must satisfy:

$$
6 \geq m_{i} \geq 2 .
$$

For the four-arm robot mechanism adopted in this paper, when the chain is folded, all the links should be located on the plane where the folding platform is located. In the robot mechanism, the joints are usually prismatic $(\mathrm{P})$ and revolute $(\mathrm{R})$ joints. The $\mathrm{R}$ joint in 


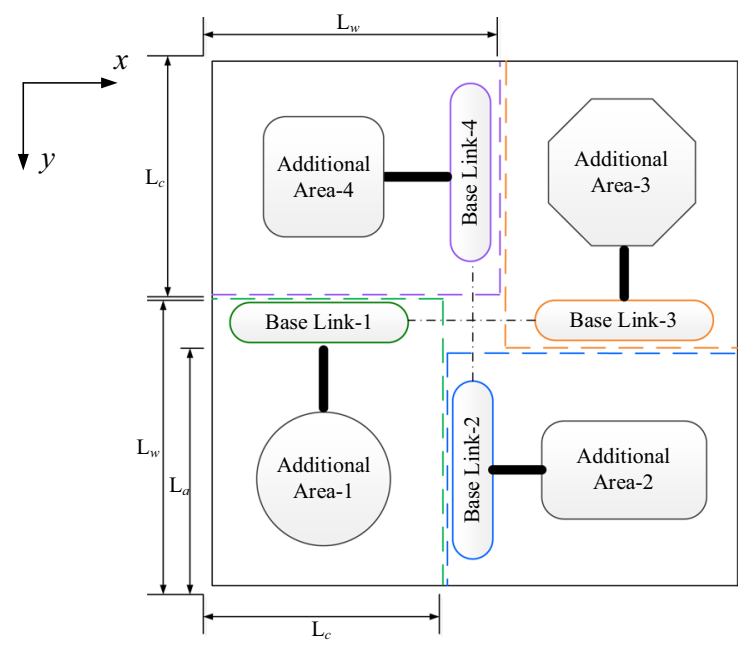

a Boundary of each storage area

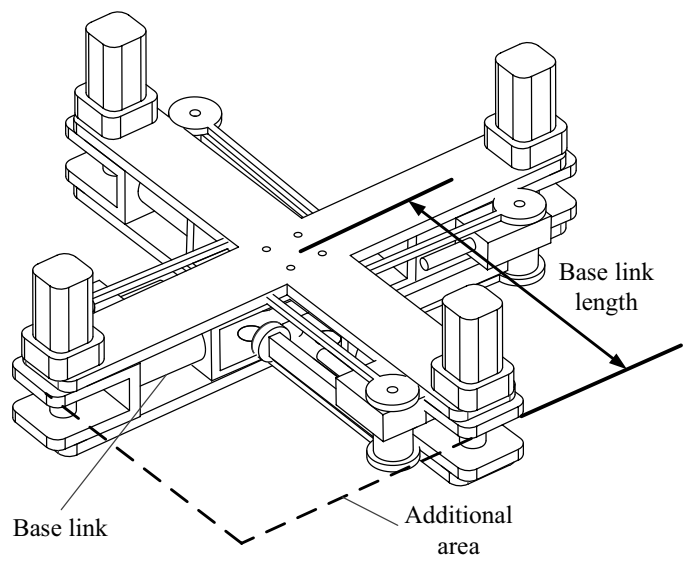

b Nonworking configuration

Figure 5 Regional arrangement of folding platform and deployable kinematic chains (DKCs)

DKC is the key joint to achieve the conversion between in-plane folded form and operation in space. The number of $\mathrm{R}$ joints should not be less than the number of $\mathrm{P}$ joints required to achieve this conversion. Therefore, the relationship between the number of $\mathrm{R}$ joints in the chain and the number of $\mathrm{P}$ joints should satisfy the following formula:

$$
R_{n} \geq P_{n} .
$$

If the number and types of joints in the chains satisfy Eqs. (4) and (5), then the DKC has the following configurations: 6R, 5R1P, 5R, 4R1P, 3R2P, 4R, 3R1P, 2R2P, 3R, 2R1P, 2R, 1R1P.

\subsection{Extensibility of DKC}

The storage region formed by the DKCs through structural characteristics is used for folding and storing the chains. The structural parameters of the chain should satisfy the constraint conditions of the storage region. As mentioned in Section 2.3, the storage area in the four-arm mechanism is the area indicated by dashed lines in Figure 6. The size of the storage area along the $x$ - and $y$-axes is set to $L_{c}$ and $L_{w}$. When the DKC is folding, the length of the longest link member in two directions in the storage area should not exceed each boundary of this area. In Figure 6, the first joint that connects to the platform is defined as the root of the $\mathrm{DKC}$, and the $\mathrm{R}$ joint at the beginning and end of each boundary is named the fold factor. Figure 6a illustrates an unreasonable configuration as the DKC exceeds the storage area. Figure $6 \mathrm{~b}$ illustrates a reasonable

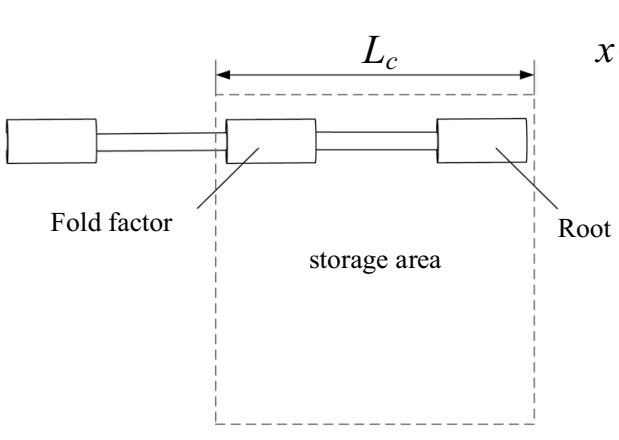

a Unreasonable configuration

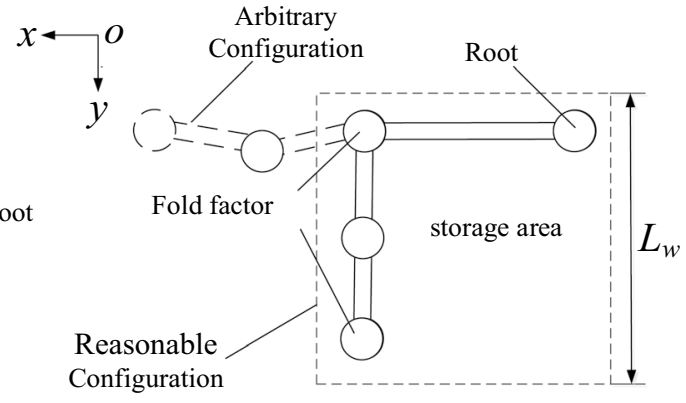

b Reasonable configuration

Figure 6 Configuration method under the restriction of storage area 
configuration. From Figure 6, we can observe that the fold factor determines whether the DKC can fold into the area reasonably.

The DKCs with deployable performance include two aspects:

(1) Each link in the kinematic chain is in the same plane when folded;

(2) Each link connected by the fold factor can rotate toward the root.

Next, we extend the study to determine how the links connect together to satisfy these two aspects. In Figure $7 \mathrm{a}$, we assume the folding movement from the first joint to the last joint of DKC follows the following direction: $a, b, c, d$. By comparing Figure $7 \mathrm{a}$ and $\mathrm{b}$, it can be observed that link BC in Figure 7a is collinear with the axis of the $\mathrm{R}$ joint, i.e., $\mathrm{B}$. The $\mathrm{R}$ joint (B) can produce a circular trajectory through its rotation; moreover, it cannot change the direction of the link BC or move toward the root of the chain; consequently, the link has no folding capacity. Therefore, link BC should be perpendicular to the axis of joint $B$. Further, the axis of joint $\mathrm{B}$ should never be collinear in the $b$ direction. This is because, if the axis of joint $B$ is collinear in the $b$ direction, links $\mathrm{BC}$ and $\mathrm{AB}$ will interfere with each other. Therefore, based on the above two principles, we can obtain the reasonable extension of DKC along four directions as shown in Figure 7c.

After determining all the fold factors of DKC, we can add $\mathrm{R}$ and $\mathrm{P}$ joints between the fold factors to obtain the DKCs with different DOFs. Here, the direction of the axis of the added $\mathrm{R}$ joint can be arbitrary.

\subsection{Base Assembly Library}

Based on the above conclusions, we can obtain different configurations of DKCs. To design different types of DKCs to operate a single arm as a multiarm robot mechanism, we can use the following steps. First, by using the equations in Section 3.1, we can determine the required number of joints $m$, which can be obtained according to the number of DOFs achieved at the end of the DKC, that is, the number of joints should be equal to the number of DOFs. Then, after the number of joints is obtained, the method proposed in Section 3.2 is used to design the deployable characteristic of the kinematic chains so that they can be stored in the specified area.

For a deployable design, we should determine the number of fold factors in the chain. As mentioned above, the fold factors are the $\mathrm{R}$ joints at the beginning and end of each boundary. For the storage area proposed in this
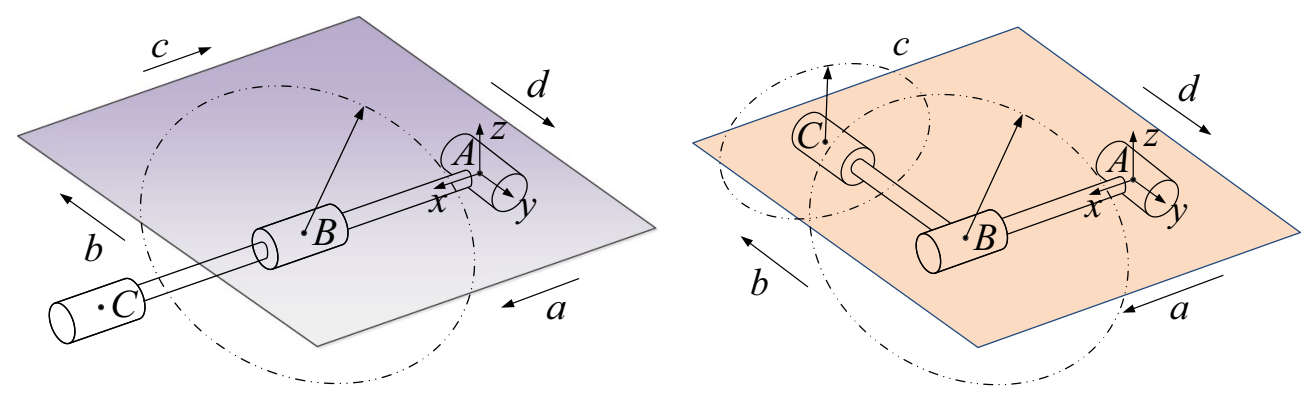

a Reasonable extension of DKCs

b Reasonable extension of DKCs
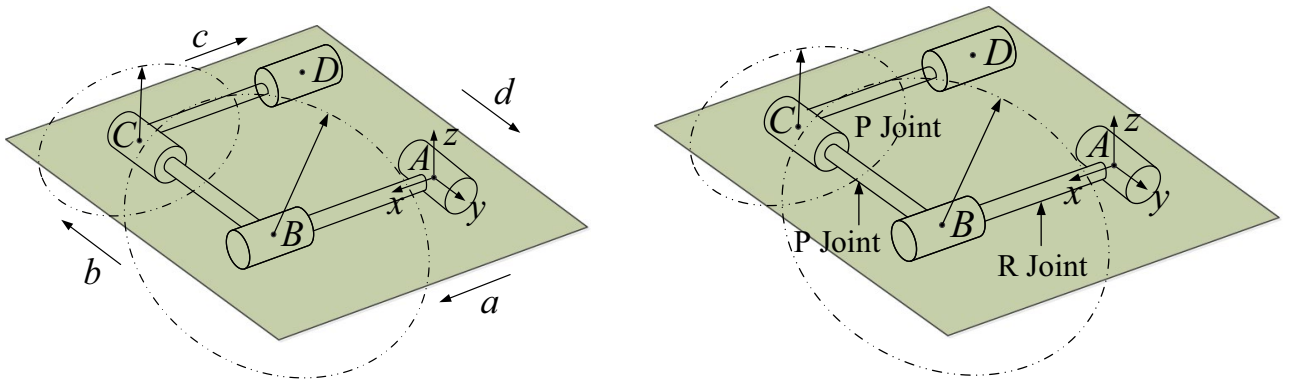

c Folding configuration of DKCs

d Add $\mathrm{P}$ and $\mathrm{R}$ joints between fold factors

Figure 7 Configuration of the DKC 
paper, the number of fold factors in DKC can be $1,2,3$, or 4, as shown in Figure 8.

The output can be designed as a P or R joint in any type according to the requirement of the connection with the object, as shown in Figure 9. Then, we can add different joints based on the configurations in Figure 9 to obtain different DKC structures with any output mode. Figure 10 shows certain cases of DKCs that contain two and three fold factors to illustrate the design procedure of the DKC.

In Figure 10, it can be observed that the axis of the first fold factor (root) is along the $y$-axis. In fact, the root axis can also be along the $x$ - or $z$-axis. The directions of the $\mathrm{R}$ joints between the folding factors can be arbitrary. Therefore, several DKC configurations can be obtained. All the obtained DKC configurations constitute the BAL. Because there are too many configurations of the BAL, this article does not enumerate all of
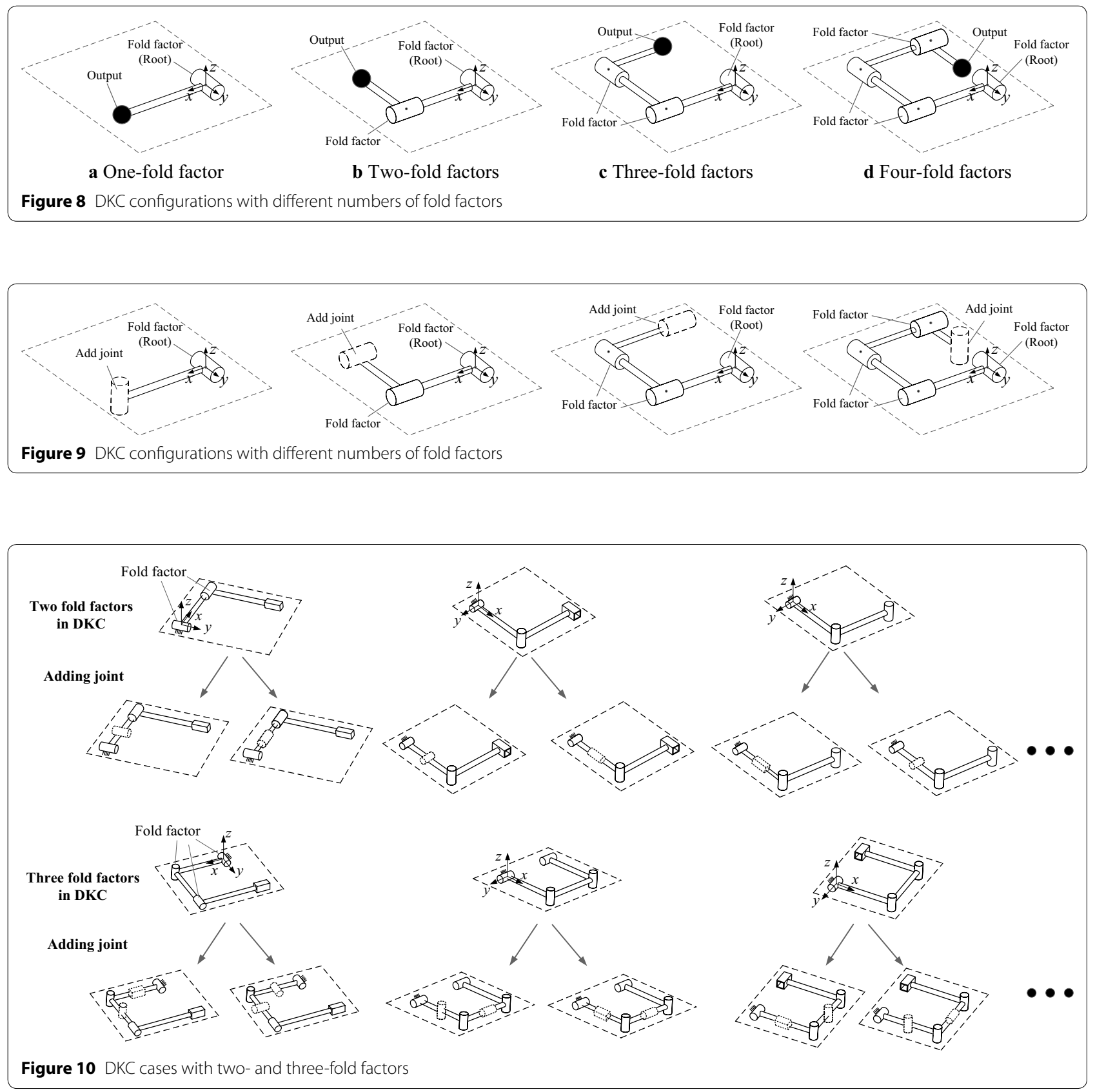
them. It should be noted that every DKC output motion can be expressed as a set [25]. After the motion set is assigned to each DKC, the appropriate DKC configuration can be selected according to the required output motion.

\section{Design of Multiarm Robot Mechanism Based on DKCs}

\subsection{Combination Design Method of Multiarm Robot Mechanism}

\subsubsection{Chain Decomposition Method}

The design method of the traditional parallel mechanism $[27,28]$ is to distribute the DOF of the end effector into each limb to obtain the ideal configuration. For a Type-I mechanism, the DKCs of the mechanism can release and connect to the object through releasable joints to achieve the transformation of the working mode. Therefore, DKC groups corresponding to different operation modes must be decomposed and represented. From Table 1, it can be noted that the number of connections between DKCs and the operation object is 2,3 , and 4 in the four-arm mechanism. In this section, we use a motion set to describe the motion of the operation object that can be achieved by connecting to the multiarm robot mechanism. The motion set can be decomposed into different subsets. Then, these subsets can be implemented through different DKCs that are connected to the object. Here, we define this method as the chain decomposition method and use the chain decomposition to describe the motion of the object connected to different DKCs.

When the number of connections between the DKCs and the operation object is two, it indicates that each working mode consists of two different DKCs connected to the object to form a parallel mechanism. There are six chain groups of two different DKCs in the four-arm mechanism.

According to Table 1, the chain groups contain two different DKCs corresponding to the case $(L=4, R=2)$ that can complete the switch of six different modes. As shown in Figure 11, the platform connects to the operation object through two DKCs, and the motion set of the object should be equal to the intersection of the motion sets of two DKCs, which can be expressed as follows.

$$
\mathrm{M}_{1}=\left\{\mathrm{S}_{1} \cap \mathrm{S}_{2}\right\}
$$

Here, $M_{i}$ represents the motion that can be achieved when the operation object is connected to the $i$-th chain group, $S_{i}$ represents the motion provided by the $i$-th chain.

Because there are six chain groups of two different DKCs in the four-arm mechanism, six motion sets of each working mode are obtained. Hence, the total motion set of the object that is generated by the mechanism can be calculated through the union of motion sets of each working mode.

$$
\begin{aligned}
\mathrm{M}= & \left\{\mathrm{M}_{1} \cap \mathrm{M}_{2} \cup \mathrm{M}_{3} \cup \mathrm{M}_{4} \cup \mathrm{M}_{5} \cup \mathrm{M}_{6}\right\} \\
= & \left\{\mathrm{S}_{1} \cap \mathrm{S}_{2}\right\} \cap\left\{\mathrm{S}_{2} \cap \mathrm{S}_{3}\right\} \cup\left\{\mathrm{S}_{3} \cap \mathrm{S}_{4}\right\} . \\
& \cup\left\{\mathrm{S}_{1} \cap \mathrm{S}_{4}\right\} \cup\left\{\mathrm{S}_{2} \cap \mathrm{S}_{4}\right\} \cup\left\{\mathrm{S}_{1} \cap \mathrm{S}_{3}\right\}
\end{aligned} .
$$

To illustrate the construction of chain decomposition, we consider the motion set of the object into two chain groups as an example. First, the motion set of the object is given as:

$$
\mathrm{M}=\left(\mathrm{T}_{u} 0 \mathrm{~T}_{w} ; 0 \mathrm{R}_{v} 0\right),
$$

where $\mathrm{T}_{i}$ represents the operation object moving along $i$ direction, $\mathrm{R}_{i}$ represents the rotation of the operation object in $i$ direction.

Let us assume that $\{\mathrm{M}\}$ is the component of motion transmission sets $M_{1}$ and $M_{2}$, that is $\left\{M_{1} \cup M_{2}\right\} \subset\{M\}$; then, we can select a special assignment $\left\{S_{1} \cap S_{3}\right\}=\left\{M_{1}\right\},\left\{S_{2} \cap S_{4}\right\}=\left\{M_{2}\right\}$; the motion transmission set $\mathrm{M}_{1}$ can be written as:

$$
\begin{aligned}
& \left\{\mathrm{M}_{1}\right\}=\left\{\mathrm{S}_{1} \cap \mathrm{S}_{3}\right\} \\
& =\left(\mathrm{T}_{u} 0 \mathrm{~T}_{w} ; 0 \begin{array}{llll}
0 & 0 & 0
\end{array}\right)^{\circ}
\end{aligned}
$$

Similarly, $\mathrm{M}_{2}$ can be written as:

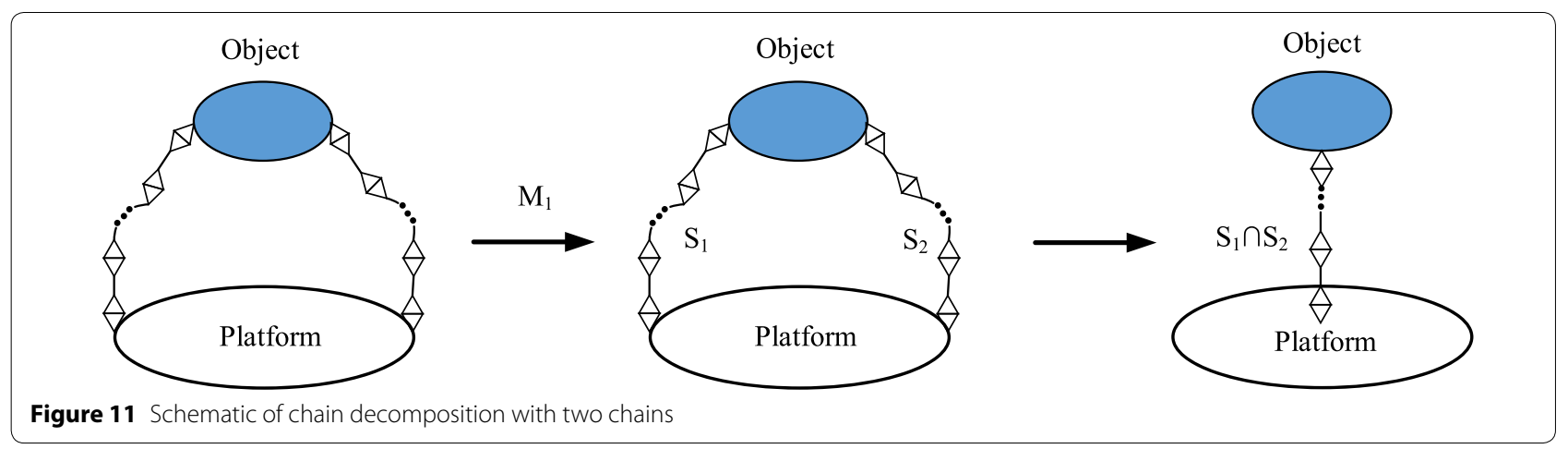




$$
\begin{aligned}
\left\{\mathrm{M}_{2}\right\} & =\left\{\mathrm{S}_{2} \cap \mathrm{S}_{4}\right\} \\
& =\left(\begin{array}{llll}
0 & 0 & \mathrm{~T}_{w} ; 0 \mathrm{R}_{v} & 0
\end{array}\right) .
\end{aligned}
$$

Then:

$$
\begin{aligned}
& M=\left\{M_{1} \cup M_{2}\right\} \\
& =\left(\mathrm{T}_{u} 0 \mathrm{~T}_{w} ; 000\right) \cup\left(\begin{array}{lllllll}
0 & 0 & 0 & 0
\end{array} \mathrm{~T}_{w} ; 0 \mathrm{R}_{v} 0\right) . \\
& =\left(\mathrm{T}_{u} 0 \mathrm{~T}_{w} ; 0 \mathrm{R}_{v} 0\right)
\end{aligned}
$$

However, this is just one of the cases that divide the motion set of the object to subsets of different chain groups. We can obtain the appropriate mechanism according to the different subsets.

Similarly, there are four chain groups of three different DKCs in the four-arm mechanism. The total motion set of the object that is generated by the mechanism can be expressed as:

$$
\begin{aligned}
M= & \left\{M_{1} \cup M_{2} \cup M_{3} \cup M_{4}\right\} \\
= & \left\{S_{1} \cap S_{2} \cap S_{3}\right\} \cup\left\{S_{2} \cap S_{3} \cap S_{4}\right\} . \\
& \cup\left\{S_{1} \cap S_{3} \cap S_{4}\right\} \cup\left\{S_{1} \cap S_{2} \cap S_{4}\right\}
\end{aligned} .
$$

As illustrated in Figure 12, the three DKCs can be selected to give the object a deterministic motion.

$$
\mathrm{M}_{1}=\left\{\mathrm{S}_{1} \cap \mathrm{S}_{2} \cap \mathrm{S}_{3}\right\} .
$$

If $\mathrm{M}=\left(\mathrm{T}_{u} \mathrm{~T}_{v} \mathrm{~T}_{w} ; \mathrm{R}_{u} \mathrm{R}_{v} 0\right)$, the four subsets of $\{\mathrm{M}\}$ can be determined according to the required mode switch. If the motion set $M_{1}$ is as follows:

$$
\begin{aligned}
\mathrm{M}_{1} & =\left\{\mathrm{S}_{1} \cap \mathrm{S}_{2} \cap \mathrm{S}_{3}\right\} \\
& =\left(\mathrm{T}_{u} 0 \mathrm{~T}_{w} ; \mathrm{R}_{u} 00 .\right.
\end{aligned} .
$$

Then, we determine the fourth DKC that can permit the elements of $\mathrm{M}_{2}, \mathrm{M}_{3}, \mathrm{M}_{4}$ to contain $\mathrm{T}_{v}$ and $\mathrm{R}_{v}$. Then, the motion set of the object is obtained by the union of the four subsets.

$$
\begin{aligned}
M & =\left\{M_{1} \cup M_{2} \cup M_{3} \cup M_{4}\right\} \\
& =\left(T_{u} T_{v} T_{w} ; R_{u} R_{v} 0\right)
\end{aligned} .
$$

When $\mathrm{L}=4$ and $\mathrm{R}=0$, there is only one configuration where the four DKCs connect to the object. Moreover, the motion set of the object is:

$$
M=\left\{S_{1} \cap S_{2} \cap S_{3} \cap S_{4}\right\} .
$$

\subsubsection{Platform Decomposition Method}

In the Type-II mechanism, there are two platforms with DKCs. The DKCs of each platform can connect to an object to form a parallel mechanism, and the working task is achieved by the concurrent operation of the formed two parallel mechanisms. Thus, the Type-II mechanism can be treated as two Type-I mechanisms fixed together. According to the task, we can determine the relative motion required to complete the motion. To complete the relative motion, the independent motion that two platforms must produce is denoted as N. Then, it is distributed to the two platforms so that each motion set contains $\mathrm{N}$. The schematic diagram of platform decomposition with three DKCs is shown in Figure 13.

As shown in Figure 13, the motion sets of object- 1 of the upper platform and object- 2 of the lower platform are denoted as $\left\{M_{U}\right\}$ and $\left\{M_{L}\right\}$, respectively.

The output motion of these two parallel mechanisms can be expressed as follows:

$$
\mathrm{M}_{\mathrm{U}}=\mathrm{S}_{\mathrm{U} 1} \cap \mathrm{S}_{\mathrm{U} 2} \cap \mathrm{S}_{\mathrm{U} 3} ; \mathrm{M}_{\mathrm{L}}=\mathrm{S}_{\mathrm{L} 1} \cap \mathrm{S}_{\mathrm{L} 2} \cap \mathrm{S}_{\mathrm{L} 3} .
$$

According to the task, we can determine the motion element for the relative motion generation. The motion element, noted by $N$, is the subset of $\left\{M_{U}\right\}$ and $\left\{M_{L}\right\}$ :

$$
\mathrm{N} \subseteq \mathrm{M}_{\mathrm{U}} ; \mathrm{N} \subseteq \mathrm{M}_{\mathrm{L}}
$$

Therefore, when the motion sets of two parallel mechanisms that are formed by the upper and lower platforms contain the motion element, each parallel mechanism can produce relatively independent motion. In Section 4.3, we will introduce a case to illustrate this design procedure.
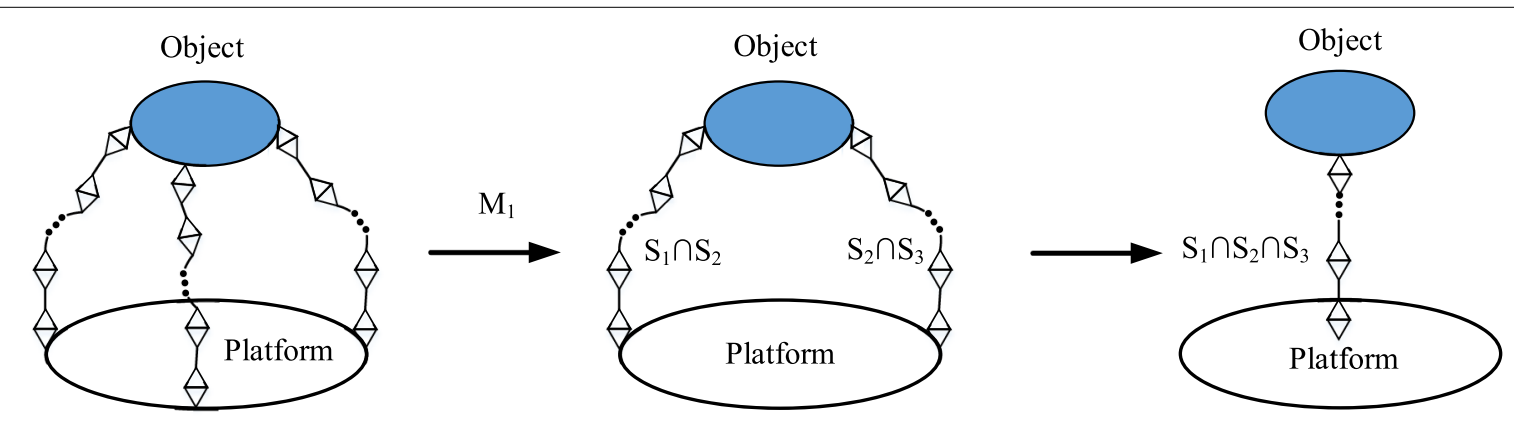

Figure 12 Schematic of chain decomposition topology with three chains 


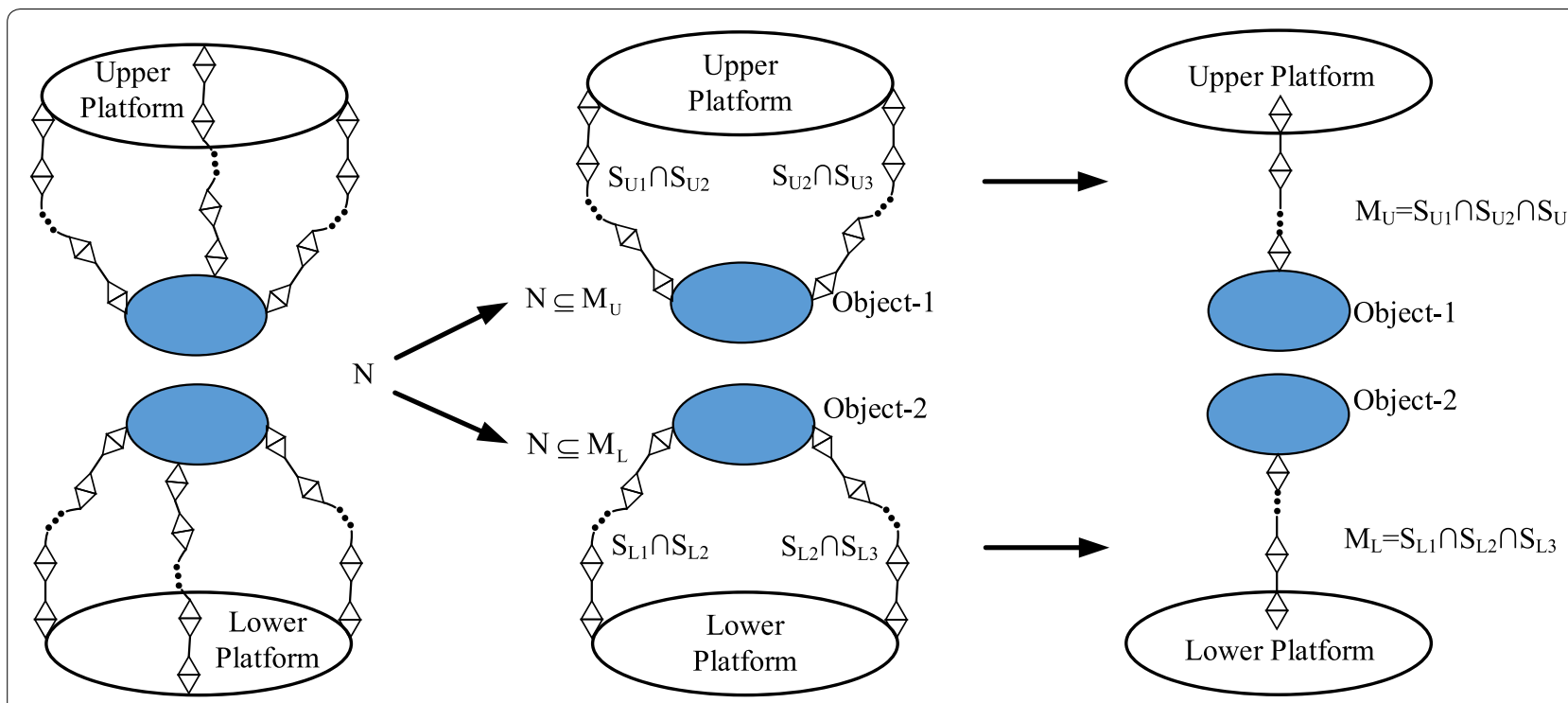

Figure 13 Schematic of platform decomposition with three DKCs

\subsection{Design Procedure of Multiarm Robot Mechanism}

If we use the attribute of the parallel mechanism structure to determine the combination scheme of DKCs, the motion set $\{M\}$ is always given first in the design procedure. After determining $\{\mathrm{M}\}$, we can determine whether the mode switch is required to complete the motion. Then, the numbers of DKCs can be obtained according to Table 1 and a further structure of the active and resting limbs can be determined. Next, we can use the formulas and the limitations of the storage area to determine the joint number and select the DKCs of the BAL that satisfy the condition. Consequently, the multiarm robot mechanism designed based on this method is intrinsically related to the DKCs and operation object, which share the same structural attributes as the parallel mechanisms designed previously by other researchers. The design procedure is illustrated in Figure 14.

\subsection{Case Study}

\subsubsection{Type-I Multiarm Robot Mechanism}

As shown in Figure 14, the first step is to determine the motion mode and whether the mode switch is required. Here, we assume that the motion set to be achieved by the mechanism is $\mathrm{M}=\left(\mathrm{T}_{u} \mathrm{~T}_{v} \mathrm{~T}_{w} ; \mathrm{R}_{u} \mathrm{R}_{v} 0\right)$ and it can be achieved by the mode switch.

Then, the second step is to determine the number of active and resting DKCs. For the four-arm mechanism in this paper, let us consider an example as $\mathrm{L}=4, \mathrm{R}=1$, that is, there are three DKCs connected to the object in each mode. Under this configuration, each mode can produce three motion types on the object. Moreover, four groups of three DKCs can produce four different motion types on the object.

The third step is to divide $\mathrm{M}$ into different groups for motion mode switch. As mentioned in the second step, four modes can achieve motion when we consider the configuration as $\mathrm{L}=4, \mathrm{R}=1$. The set corresponding to each mode is: $M_{1}, M_{2}, M_{3}, M_{4}$. Here, we consider one of the solutions as:

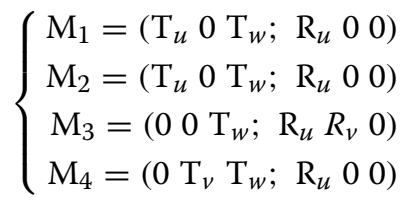

According to Eq. (19), we can select three DKCs that can satisfy $M_{1}$. That is, the intersection of the motion set $\mathrm{S}_{i}(i=1,2,3)$ of three DKCs can achieve $\mathrm{M}_{1}$. Then, a fourth DKC can be added to ensure that the remaining three chain groups satisfy the conditions of $M_{2}, M_{3}, M_{4}$. Thus, by combining the four DKCs, the four-arm mechanism can be obtained.

Figure 15 is a case of the Type-I mechanism. The mechanism can switch different working modes through different DKC groups connected to an object.

\subsubsection{Type-II Multiarm Robot Mechanism}

As shown in Figure 2, there are two platforms in the Type-II mechanism. Each platform has four DKCs, which can connect to an object to form a parallel mechanism. The Type-II mechanism can be treated as two TypeI mechanisms performing collaborative work tasks. The motion set achieved by the Type-II mechanism is 


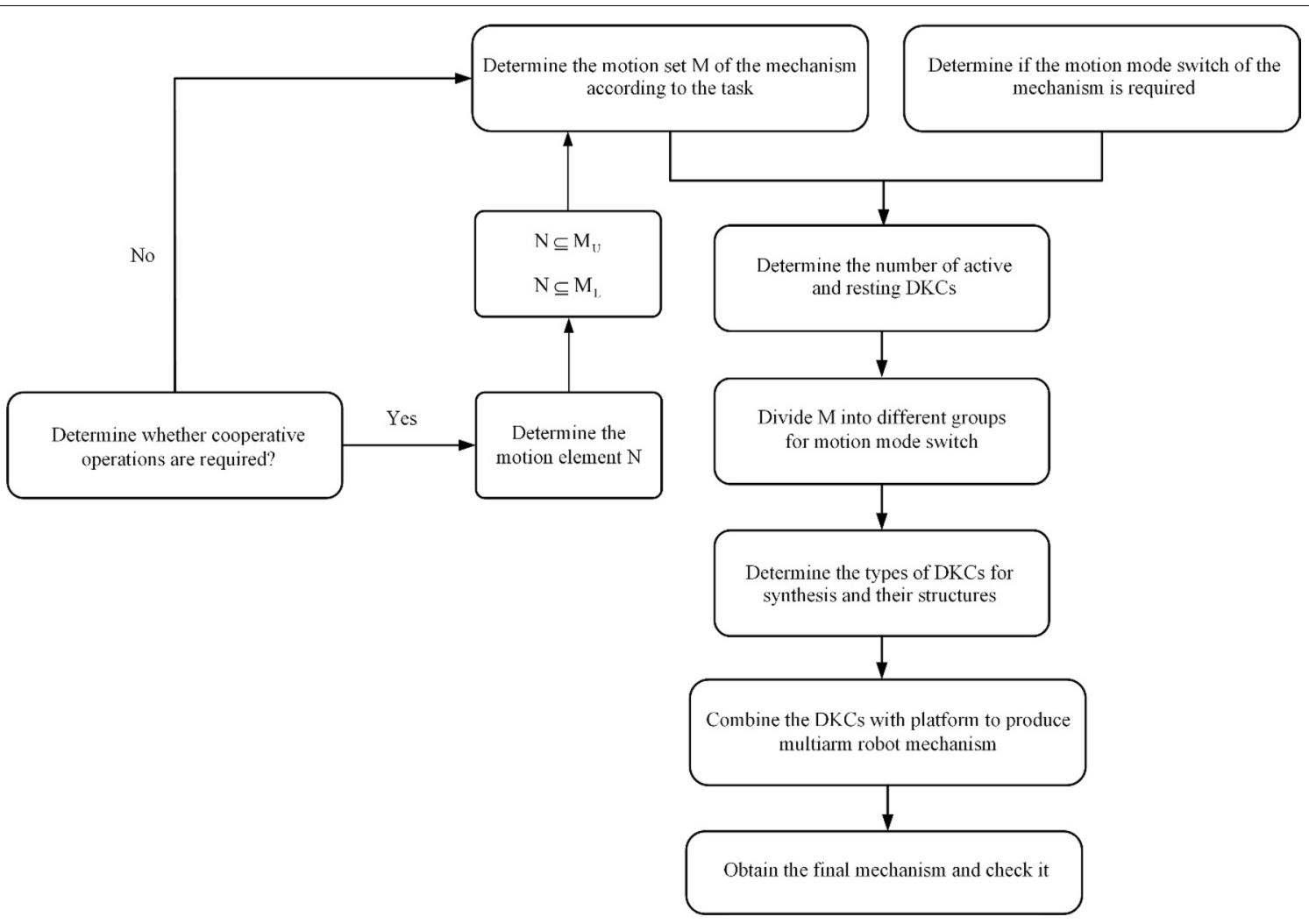

Figure 14 Design procedure of multiarm robot mechanism

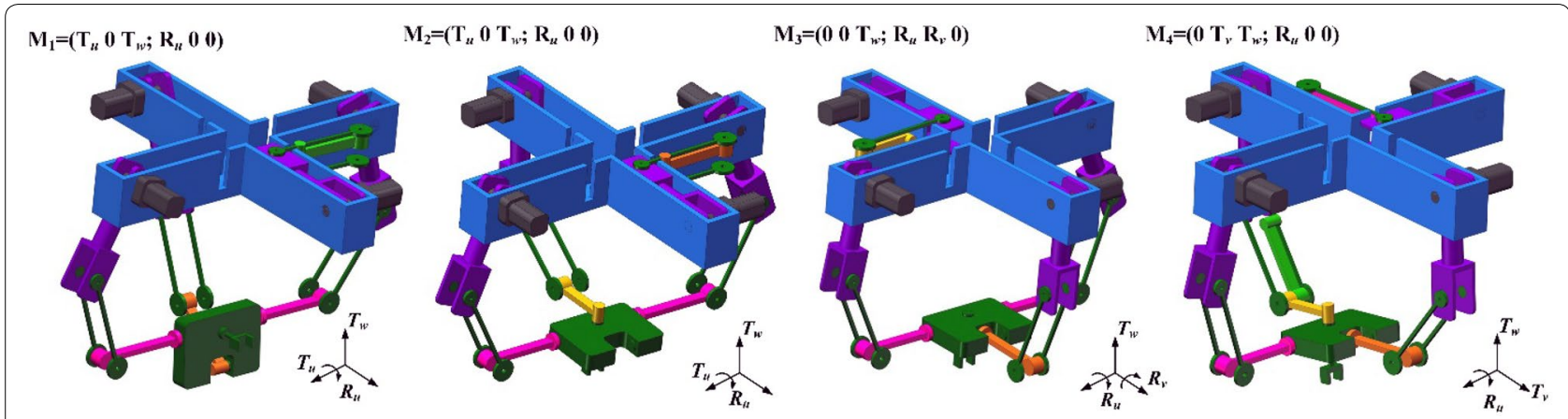

Figure 15 Four working modes of the Type-I mechanism

generated by the relatively independent motion of the two Type-I mechanisms. According to the platform decomposition method in Section 4.1.2, we can determine the key motion elements that can achieve the work task and determine the Type-I motion sets that contain the key elements. Figure 16 shows a simple bending diagram. The parallel mechanisms formed by the two platforms cooperate to achieve the bending of the plate. As can be observed from the figure, plate bending requires two platforms to achieve rotation around the $x$-axis, that is, the motion of $R_{x}$ should be included in the motion sets of upper and lower platforms.

Hence, there are several solutions to this simple bending. The motion sets generated by the upper and lower platforms must contain $\mathrm{R}_{x}$ and $\mathrm{T}_{z}$. Here, $\mathrm{N}$ is a set

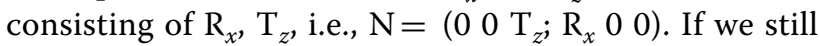
set $\mathrm{L}=4$ and $\mathrm{R}=1$, the formed two mechanisms have four operation modes, and only one of the modes is required to achieve the movement of $\mathrm{R}_{x}, \mathrm{~T}_{z}$. 

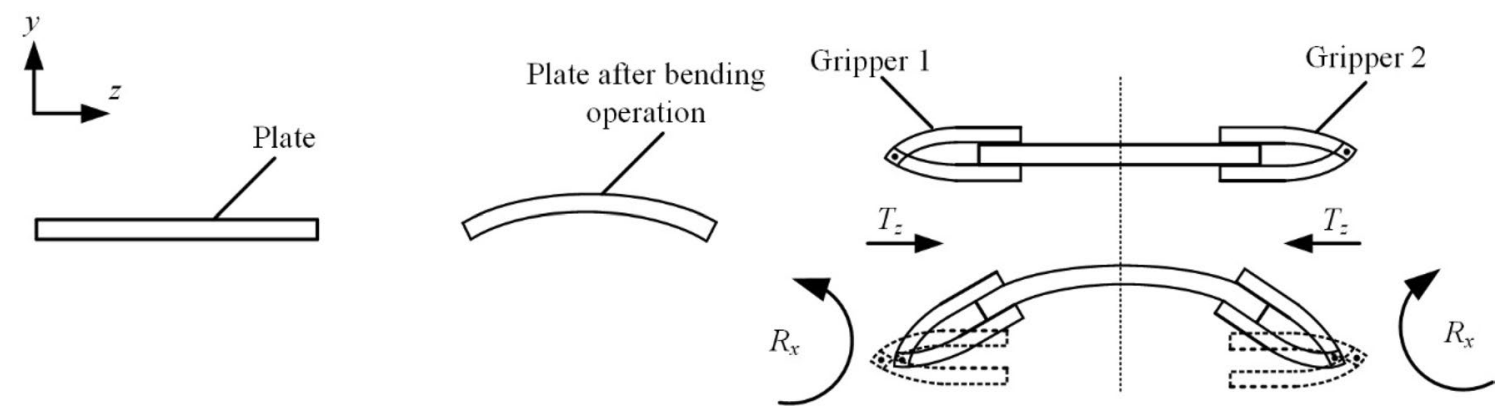

Figure 16 Diagram of the plate bending operation
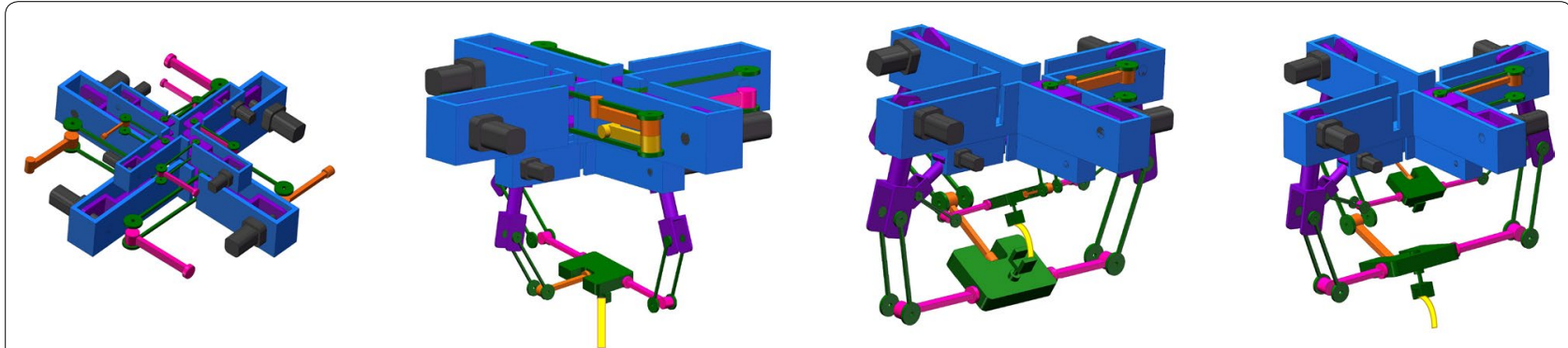

a Mechanism in nonworking state

b Grasping of the plate by the lower platform

c Plate bending

d Plate placement

Figure 17 Working modes of Type-II mechanism

Then, we can provide arbitrary values to $M_{U}$ and $M_{L}$ as long as they satisfy Eq. (18). For the convenience of further discussion, we used the aforementioned Type-I mechanism as the mechanism in the upper and lower platforms of the Type-II mechanism. The schematic diagram of this work is as shown in Figure 17.

The Type-II mechanism shown in Figure 17 is just one configuration of solutions. Through the cooperation of the two platforms, the mechanism can achieve working processes from grasping and bending to placing. We can adjust the values of $\mathrm{M}_{\mathrm{L}}$ and $\mathrm{M}_{\mathrm{U}}$ according to different requirements to achieve the given task. Different $M_{L}$ and $\mathrm{M}_{\mathrm{U}}$ sets can obtain different combinations of DKCs. In future work, we will study the total number of configurations that can be obtained to form a unified calculation method.

\subsection{Fold-Extension Ratio}

Figure 18 shows three DKCs that are extended to connect to an object. It is interesting to determine the benefits of the multiarm robot mechanism in deployable operations. In Figure 18, the structure of each DKC is the same except the releasable joint at the end. Next, we will study the relationship between the reachable space of DKC and the size of the mechanism in folding.

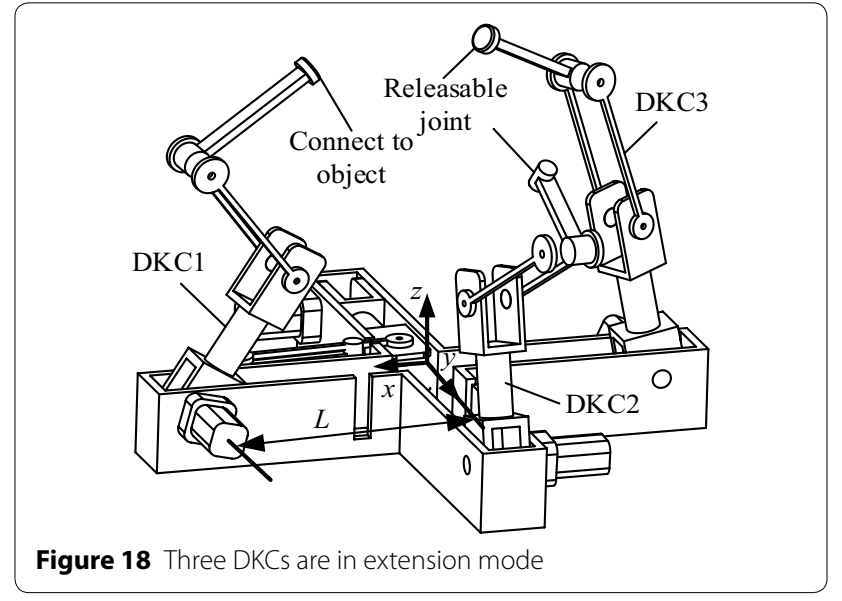

The coordinate system $O-x y z$ is established at the center of the platform as shown in Figure 18. The distance between point $O$ and the axle of the first joint is $L$. The structure parameters of DKC are shown in Figure 19. In $\mathrm{DKC} 1$, while using the $\mathrm{D}-\mathrm{H}$ coordinate transformation method, each D-H parameter is as listed in Table 2.

According to Figure 19, the transformation matrix from point $O$ to releasable joint is: 


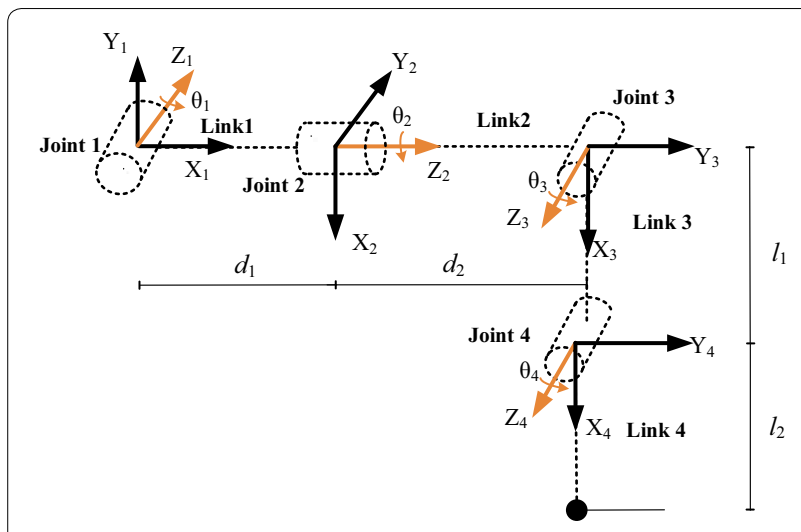

Figure 19 Diagram of the DKC structure

Table 2 D-H parameters

\begin{tabular}{lllll}
\hline Joint $\boldsymbol{i}$ & $\boldsymbol{a}_{\boldsymbol{i}}$ & $\boldsymbol{a}_{\boldsymbol{i}}$ & $\boldsymbol{d}_{\boldsymbol{i}}$ & $\boldsymbol{\theta}_{\boldsymbol{i}}$ \\
\hline 1 & $d_{1}$ & 90 & 0 & $\theta_{1}$ \\
2 & 0 & 90 & $d_{2}$ & $\theta_{2}$ \\
3 & 0 & 0 & $l_{1}$ & $\theta_{3}$ \\
4 & 0 & 0 & $I_{2}$ & $\theta_{4}$ \\
\hline
\end{tabular}

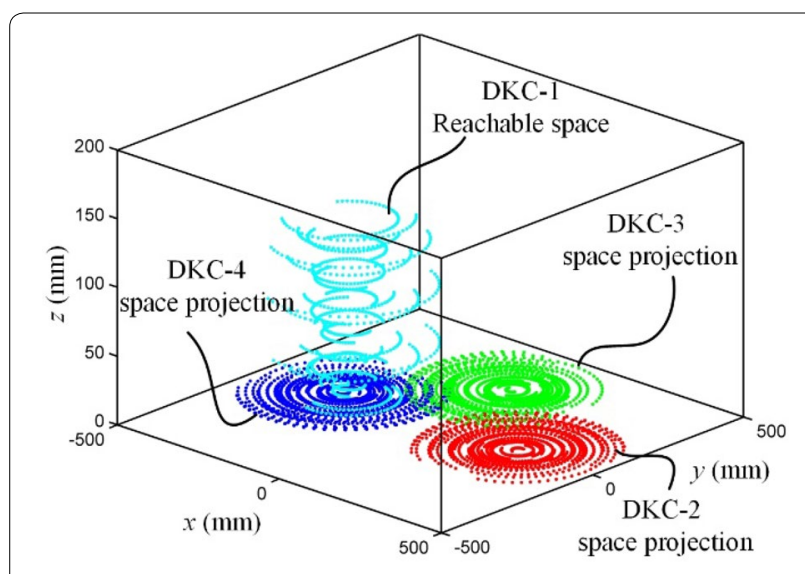

Figure $\mathbf{2 0}$ Reachable spaces of four-arm mechanism

$$
\mathrm{T}=\mathrm{T}_{1}^{O} \mathrm{~T}_{R}^{1} \mathrm{~T}_{2}^{1} \mathrm{~T}_{3}^{2} \mathrm{~T}_{4}^{3} \mathrm{~T}_{R}^{4},
$$

where $T_{n}^{m}$ is transformation matrix from $m$ to $n$.

The parameters are given as: $L=100, d_{1}=100, d_{2}=100$, $l_{1}=100, l_{2}=100$ (unit: $\mathrm{mm}$ ). The range of each joint is: $\theta_{1}$ $[45,90], \theta_{2}[-180,180], \theta_{3}[-90,90], \theta_{4}[-90,90]$ (unit: degree). Then, the reachable space of DKC1 is shown in Figure 20. Similarly, we can obtain the reachable spaces of the other three DKCs. To form a contrast with the size of the mechanism in the folding operation, the reachable

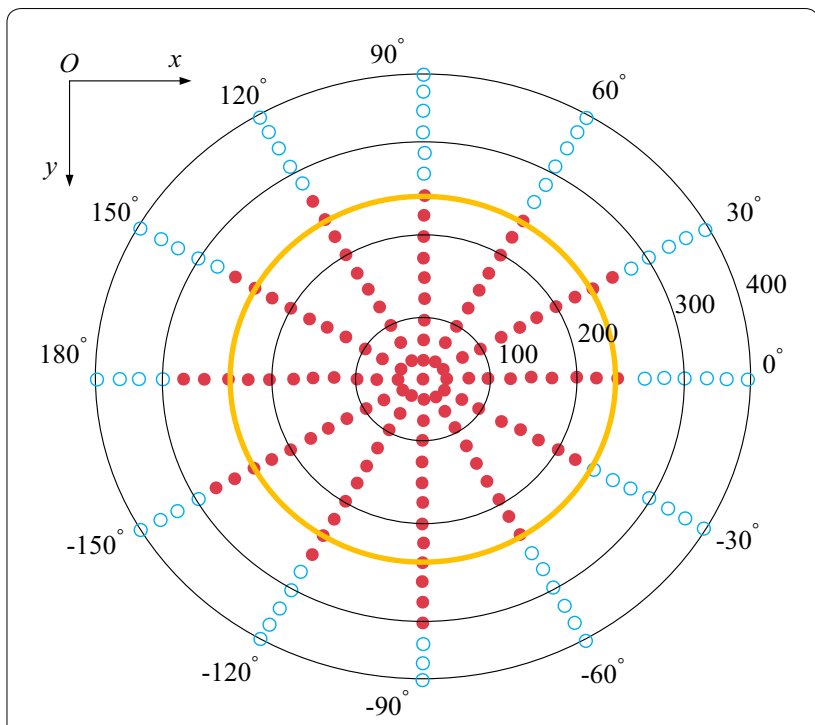

Figure $\mathbf{2 1}$ Top view of the reachable space projection

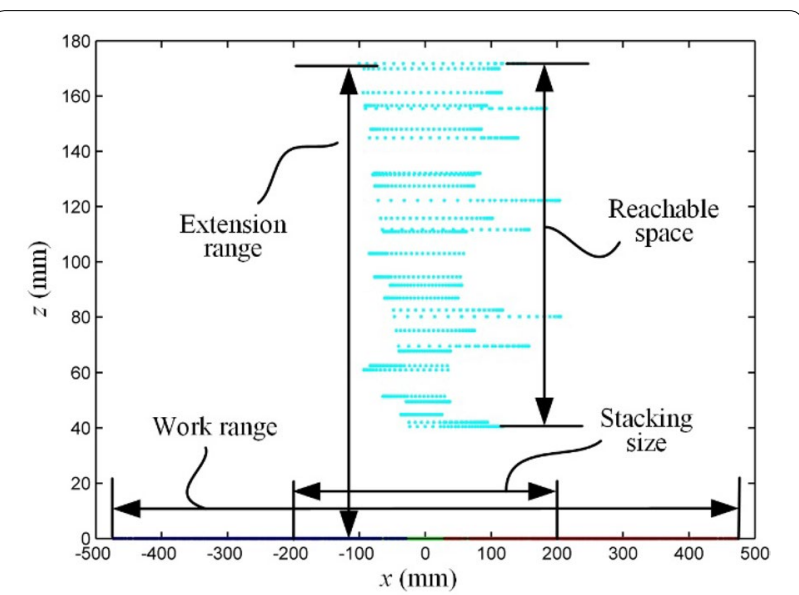

Figure 22 Fold-extension ratio diagram of the multiarm robot

spaces of DKCs-2, 3, 4 are projected to the plane $X=0$ and $Y=0$, and the result is shown in the Figure 20.

Figure 21 is the top view of the reachable space projection. In this figure, the red points are the points in reachable space. The black circle is the measuring circle with $r=100,200,300,400 \mathrm{~mm}$. The yellow circle is the effective circle, and its radius is the distance between the minimum reachable point and the center point.

For the traditional deployable mechanism, the foldextension ratio is an evaluation index. While considering the deployable mechanism proposed in this paper, its deployable operation primarily enables the multiarm to have the motions of extension and folding. Therefore, the comparison between the reachable space of the DKC and 
the size of the mechanism in the folding state can be used as the evaluation index of the fold-extension ratio of the mechanism proposed in this paper.

Figure 22 is the fold-extension ratio of the multiarmed robot. The stacking size is the size occupied by the mechanism itself in the folding state, that is, the distance between the axis of the first joint of DKC1 and DKC3. The working range is the distance between the boundaries of the effective circles of DKC2 and DKC4. As it can be observed in Figure 22, the range of stacking size is [-200, 200]; therefore, the length is $400 \mathrm{~mm}$. The working range is [-480, 480]; therefore, its length is 960 $\mathrm{mm}$. The ratio of stacking size to the work range is 1:2.4. Therefore, the fold-extension ratio is equal to 1:2.4. In the longitudinal direction, the mechanism can expand from 0 to $180 \mathrm{~mm}$. Consequently, the mechanism has a good deployable performance. It can certainly optimize the occupied volume during transportation and has multifunction operation performance.

\section{Conclusions}

1) A methodology for the design of the multiarm robot mechanisms with DKCs was presented. The principle of construction was demonstrated by combining the DKCs and operation. The operation mode can be switched based on the DKCs and self-assembly can be performed.

2) A series of DKCs were obtained by the constraint conditions of the designated area and the DOFs of the single-arm mechanism. The DKC is a good choice to design the multiarm robot mechanisms with certain advantages.

3) Type-I and Type-II robot mechanisms with four arms were obtained based on the combination design method. The proposed multiarm robot mechanisms retain the original functions of the traditional multiarm robot mechanisms; moreover, it demonstrates the exclusive performance of the DKCs.

4) Fold-extension ratio was discussed and calculated, and the result demonstrated that the mechanism can optimize the occupied volume during transportation and has multifunction operation performance.

\section{Acknowledgements}

Not applicable.

\section{Authors' Contributions}

SG was in charge of the whole trial; FZ wrote the manuscript; HS assisted with design and analyses. HQ and $\mathrm{YC}$ assisted with concept design. All authors read and approved the final manuscript.

\section{Authors' Information}

Fu-Qun Zhao, born in 1992, is currently a PhD candidate at School of Mechanical, Electronic and Control Engineering, Beijing Jiaotong University, China. He received his master degree from Beijing Jiaotong University, China, in 2016. His research interests include robot mechanism design, kinematics analysis.

Sheng Guo, born in 1972, is currently a professor at School of Mechanical, Electronic and Control Engineering, Beijing Jiaotong University, China. His main research interests include spatial mechanism design, parallel robot and medical rehabilitation robot.

Hai-Jun Su, born in 1973, is currently a professor at Department of Mechanical and Aerospace Engineering, The Ohio State University, Columbus, USA. His research interests include robotic, complaint mechanism, DNA origami mechanism.

Hai-Bo Qu, born in 1983, is currently an associate professor at Robotics Institute, Beijing Jiaotong University, China. He received his PhD from Beijing Jiaotong University in 2013. His research interests include robotics mechanism and mechanical design.

Ya-Qiong Chen, born in 1983, is currently an experimentalist at Robotics Institute, Beijing Jiaotong University, China. He received his PhD from Beijing Jiaotong University in 2017. His research interests include robotics mechanism and mechanical design.

\section{Funding}

Supported by National Natural Science Foundation of China (Grant No. 51875033), the Fundamental Research Funds for the Central Universities (Grant No. 2018JBM051), program of China Scholarships Council (Grant No. CSC201907090037).

\section{Competing interests}

The authors declare no competing financial interests.

\section{Author Details}

${ }^{1}$ School of Mechanical, Electronic and Control Engineering, Beijing Jiaotong University, Beijing 100044, China. ${ }^{2}$ Department of Mechanical and Aerospace Engineering, The Ohio State University, Columbus, OH 43210, USA.

Received: 31 January 2020 Revised: 9 September 2020 Accepted: 6 October 2020

Published online: 28 October 2020

\section{References}

[1] X L Ding, F Yang. Study on hexapod robot manipulation using legs. Robotica, 2016, 34(2): 468-481.

[2] J Seo, M Yim, and V Kumar, A theory on grasping objects using effectors with curved contact surfaces and its application to whole-arm grasping. The International Journal of Robotics Research, 2016, 35(9): 1080-1102.

[3] D Stonge, I Sharf, L Sagnières, et al. A deployable mechanism concept for the collection of small-to-medium-size space debris. Advances in Space Research, 2017, 61(5): 1286-1297.

[4] Y Xu, F Gao, Y Pan, et al. Method for six-legged robot stepping on obstacles by indirect force estimation. Chinese Journal of Mechanical Engineering, 2016, 29(4): 669-679.

[5] N Rojas, A M Dollar. Gross motion analysis of Fingertip-Based Within-Hand manipulation. IEEE Transactions on Robotics, 2016, 32(4):1009-1016.

[6] J Liu, X Zhang, K Zhang, et al. Configuration analysis of a reconfigurable Rubik's snake robot. Proceedings of the Institution of Mechanical Engineers, Part C: Journal of Mechanical Engineering Science, 2019, 233(9): 3137-3154.

[7] W Xu, J Peng, B Liang, et al. Hybrid modeling and analysis method for dynamic coupling of space robots. IEEE Transactions on Aerospace and Electronic Systems, 2016, 52(1): 85-98.

[8] X Zhang, J Liu, J Feng, et al. Effective capture of nongraspable objects for space robots using geometric cage pairs. IEEE Transactions on Mechatronics, 2020, 25(1): 95-107.

[9] J Liu, X Zhang, G Hao. Survey on research and development of reconfigurable modular robots. Advances in Mechanical Engineering, 2016, 8(8): $1-21$ 
[10] E Özgür, G Gogu, Y Mezouar. A study on dexterous grasps via parallel manipulation analogy. Journal of Intelligent and Robotic Systems, 2017, 87: $1-12$.

[11] E Özgür, G Gogu, Y Mezouar. Structural synthesis of dexterous hands[C]. IEEE/RSJ International Conference on Intelligent Robots and Systems, Chicago, USA, September 14-18, 2014: 1676-1681.

[12] J Borràs, A Dollar. Analyzing dexterous hands using a parallel robots framework. Auton Robot, 2014, 36: 169-180.

[13] J Borràs, A Dollar. Framework comparison between a multi-fingered hand and a parallel manipulator. Computational Kinematics, 2014, 15: 219-227.

[14] X Ding, Y Zheng, KXu. Wheel-legged hexapod robots: a multifunctional mobile manipulating platform. Chinese Journal of Mechanical Engineering, 2017, 30(1): 3-6.

[15] Y Zhai, P Gao, Y Sun, et al. Gait planning for a multi-motion mode wheellegged hexapod robot. IEEE International Conference on Robotics and Biomimetics, Qingdao, China, December 3-7, 2016: 449-454

[16] B Corves, T Mannheim, M Riedel. Re-grasping: Improving capability for multi-arm robot system by dynamic reconfiguration. International Conference on Intelligent Robotics and Applications, Aachen, Germany, December 6-8, 2011: 132-141.

[17] M Nefzi, M Riedel, B Corves. Development and design of a multi-fingered gripper for dexterous manipulation. IFAC Proceedings Volumes, 2006, 39(16): 133-138.

[18] N Koyachi, T Arai, H Adachi, et al. Hexapod with Integrated Limb Mechanism of Leg and Arm. Proceedings of the IEEE International Conference on Robotics and Automation, Nagoya, Aichi, Japan, May 21-27, 1995: 1952-1957.

[19] K Inoue, Y Nishihama, T Arai, et al. Mobile Manipulation of Humanoid Robots-Body and Leg Control for Dual Arm Manipulation. Proceedings of the IEEE International Conference on Robotics and Automation, Washington, DC, USA, May 11-15, 2002: 2259-2264.
[20] L Yan, H Yuan, W Xu, et al. Generalized relative Jacobian matrix of space robot for dual-arm coordinated capture. Journal of Guidance Control and Dynamics, 2018, 41(5):1-7.

[21] J Kindracki, KTur, P Paszkiewicz, et al. Experimental research on low-cost cold gas propulsion for a space robot platform. Aerospace science and technology, 2017, 62(1): 148-157.

[22] P Huang, M Wang, Z Meng, et al. Reconfigurable spacecraft attitude takeover control in post-capture of target by space manipulators. Journal of the Franklin Institute, 2016, 353(9): 1985-2008.

[23] H Park and C Lee. Dual-arm coordinated-motion task specification and performance evaluation. IEEE/RSJ International Conference on Intelligent Robots and Systems, Daejeon, South Korea, Oct 9-14, 2016: 929-936.

[24] W Cao, Z Jing, H Ding. A general method for kinematics analysis of two-layer and two-loop deployable linkages with coupling chains[J]. Mechanism and Machine Theory, 2020, 152(1): 103945.

[25] D St-Onge, C Gosselin. Synthesis and design of a one degree-of-freedom planar deployable mechanism with a large expansion ratio. Journal of Mechanisms and Robotics, 2016, 8(2): 021025.

[26] S Lu, D Zlatanov, X L Ding, et al. Novel Deployable Mechanisms With Decoupled Degrees-of-Freedom. Journal of Mechanisms and Robotics, 2016, 8(2): 021008.

[27] J He, F Gao, X Meng, et al. Type synthesis for 4-DOF parallel press mechanism using $G_{F}$ set theory. Chinese Journal of Mechanical Engineering, 2015, 28(4): 851-859.

[28] F Gao, W Li, X Zhao, et al. New kinematic structures for 2-, 3-, 4-, and 5-DOF parallel manipulator designs. Mechanism and Machine Theory, 2002, 37(11): 1395-1411.

\section{Submit your manuscript to a SpringerOpen ${ }^{\odot}$ journal and benefit from:}

- Convenient online submission

- Rigorous peer review

- Open access: articles freely available online

- High visibility within the field

- Retaining the copyright to your article

Submit your next manuscript at $\boldsymbol{\nabla}$ springeropen.com 